\title{
Development of the ability to produce secondary metabolites in Streptomyces through the acquisition of erythromycin resistance
}

\author{
Yu Imai ${ }^{1}$, Tatsuya Fujiwara ${ }^{2}$, Kozo Ochi ${ }^{3}$ and Takeshi Hosaka ${ }^{4}$ \\ The Journal of Antibiotics (2012) 65, 323-326; doi:10.1038/ja.2012.16; published online 11 April 2012
}

Keywords: antibiotic production; erythromycin resistance; secondary metabolites; Streptomyces

\begin{abstract}
Members of the genus Streptomyces are gram-positive filamentous bacteria that produce various secondary metabolites, which are exemplified by antibiotics. Because more than two-thirds of clinically useful antibiotics are produced from Streptomyces, ${ }^{1}$ members of this genus are of high pharmacological and industrial interest. Additionally, recent genome-sequencing projects have revealed that many biosynthetic gene clusters, producing unknown secondary metabolites, exist in Streptomyces genomes. ${ }^{2-4}$ The exploitation of the genomic potential of Streptomyces may lead to the isolation of new biologically active compounds. ${ }^{5,6}$ Therefore, it is extremely important for antibiotic discovery research to investigate their unexploited abilities to produce secondary metabolites and to clarify their activation mechanism. We previously demonstrated a method for activating unexpressed or poorly expressed Streptomyces genes to synthesize secondary metabolites through a mutation that confers resistance to drugs targeting the RNA polymerase and/or ribosomes. ${ }^{7-9}$ This led to the discovery of piperidamycin, a novel antibiotic produced by Streptomyces sp. 631689, which rarely produces antibiotics in any type of culture media. ${ }^{10}$ Here, we describe a new technique for advanced utilization of the abovementioned method to take complete advantage of the ability of Streptomyces to produce secondary metabolites.

Erythromycin is a macrolide antibiotic that inhibits protein synthesis by binding to the bacterial $50 \mathrm{~S}$ ribosomal subunit. Previous studies have demonstrated that generating a mutation that confers resistance to a drug, such as rifampicin, streptomycin, gentamicin, paromomycin or thiostrepton, is an effective approach to increasing the production of the blue-pigmented antibiotic actinorhodin (ACT) in Streptomyces coelicolor $\mathrm{A} 3(2)$ and Streptomyces lividans. ${ }^{911-13}$ S. coelicolor A3(2) and S. lividans are well-characterized strains of Streptomyces from a physiological and genetic viewpoint; however, the effects of an erythromycin resistance mutation on antibiotic
\end{abstract}

production in S. coelicolor A3(2) and S. lividans have not been assessed. Therefore, we examined whether the acquisition of erythromycin resistance enables S. coelicolor A3(2) and S. lividans to overproduce antibiotics.

We isolated 259 and 300 spontaneous erythromycin-resistant $\left(\mathrm{Ery}^{\mathrm{r}}\right)$ mutants of S. coelicolor $\mathrm{A} 3(2)$ strain $1147\left(\mathrm{SCP}^{+}, \mathrm{SCP}_{2}^{+}\right.$, prototroph) and S. lividans strain 1326 (S. lividans 66, SLP2 ${ }^{+}, \mathrm{SLP}^{+}$, prototroph), respectively, from colonies that grew within 4 weeks after the spores (approximately $10^{11}$ spores) were spread on GYM agar plates containing 170 or $420 \mathrm{\mu g} \mathrm{ml}^{-1}$ of erythromycin, which corresponds to approximately 3 - to 14 -fold amount of minimum inhibitory concentration. These spontaneous Ery ${ }^{\mathrm{r}}$ mutants were first characterized for the production of ACT and levels of resistance to erythromycin. The mutants were cultured using GYM, R4, R4C or modified R5 (MR5) agar medium, which revealed that 22 and $20 \%$ of spontaneous Ery ${ }^{\mathrm{r}}$ mutants isolated from strains 1147 and 1326, respectively, exhibited improved ACT production (Supplementary Table S1). Table 1 and Figures $1 \mathrm{a}$ and $\mathrm{b}$ show the characteristics of the Ery ${ }^{\mathrm{r}}$ mutants of S. coelicolor A3(2) 1147 and S. lividans 1326 that have a representative phenotype for ACT and undecylprodigiosin (RED) production or erythromycin resistance. In S. coelicolor A3(2), as in the Ery $^{\mathrm{r}}$ SCE71 and SCE234 mutants, the level of resistance to erythromycin in the high-level ACT-producing strains ranged from 200 to $400 \mathrm{~g} \mathrm{ml}^{-1}$, whereas ACT productivity in strain SCE166, which exhibited a high-level of resistance to erythromycin $\left(>500 \mu \mathrm{g} \mathrm{ml}^{-1}\right)$, was on similar level as in the parent strain 1147. Strain SCE234 was the highest antibiotic-producing Ery ${ }^{\mathrm{r}}$ mutant tested in liquid culture, and the strain demonstrated increased productivity of 22- and 4.5fold (ACT and RED, respectively). As expected, transcription analysis by RT-PCR showed that expressions of the actII-ORF4, redD and redZ genes, encoding the pathway-specific positive regulatory protein for the biosynthesis of ACT and RED, respectively, were clearly enhanced

${ }^{1}$ Interdisciplinary Graduate School of Science and Technology, Shinshu University, Nagano, Japan; ${ }^{2}$ Graduate School of Agriculture, Shinshu University, Nagano, Japan; ${ }^{3}$ Department of Health Science, Faculty of Applied Information Science, Hiroshima Institute of Technology, Hiroshima, Japan and ${ }^{4}$ International Young Researchers Empowerment Center, Shinshu University, Nagano, Japan

Correspondence: Dr T Hosaka, International Young Researchers Empowerment Center, Shinshu University, 8304 Minamiminowa, Nagano, 399-4598, Japan. E-mail: thosaka@shinshu-u.ac.jp

Received 26 August 2011; revised 24 January 2012; accepted 21 February 2012; published online 11 April 2012 
Table 1 Characteristics of spontaneous Eryr mutants isolated from S. coelicolor A3(2) and S. lividans

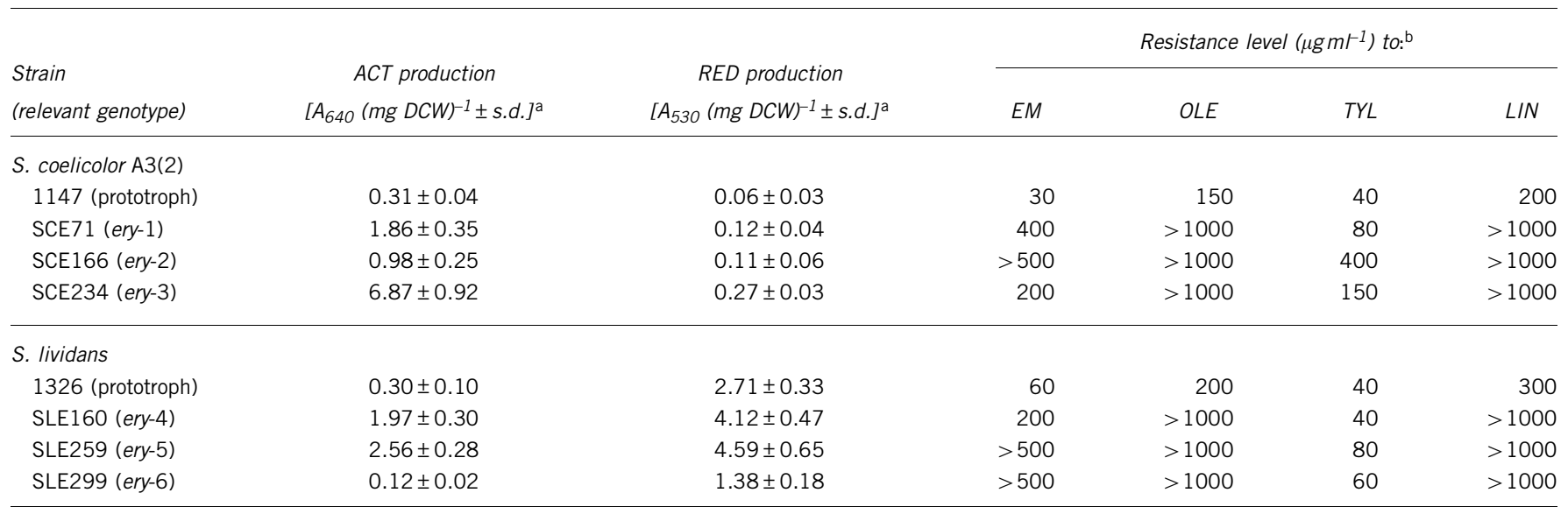

Abbreviations: ACT, actinorhodin; EM, erythromycin; LIN, lincomycin; OLE, oleandomycin; RED, undecylprodigiosin; TYL, tylosin.

aStrains were inoculated into GYM [for S. coelicolor A3(2)] or R4 (for S. lividans) liquid medium and incubated on a rotary shaker (200 r.p.m.) at $30^{\circ} \mathrm{C}$ for 8 days. ACT and RED production was determined spectrophotometrically as described by Kieser T. et al. ${ }^{1}$ The values are expressed as the mean \pm s.d. of triplicate or more samples.

${ }^{b}$ Determined after incubation on GYM agar medium at $30^{\circ} \mathrm{C}$ for 3 days.
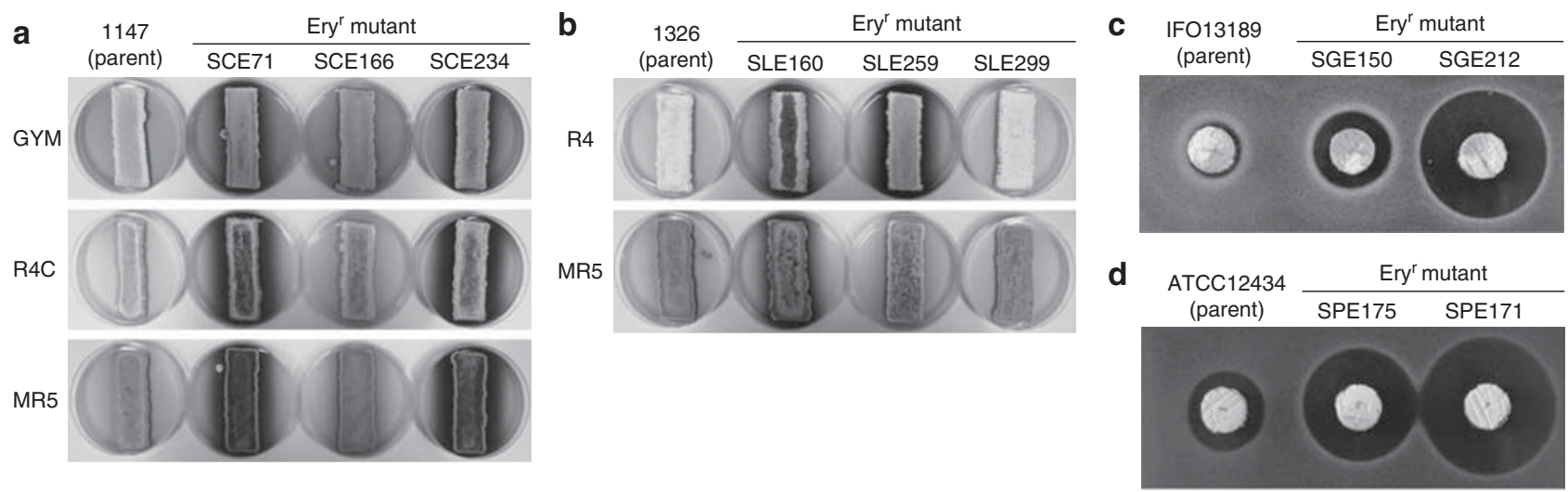

Figure 1 Activation of antibiotic production in Streptomyces spp. through the acquisition of erythromycin resistance. ACT production by parent and Ery mutant strains of S. coelicolor A3(2) 1147 (a) and S. lividans 1326 (b). Strains were inoculated onto GYM, R4C, MR5 or R4 agar medium and incubated at $30^{\circ} \mathrm{C}$ for 7 days. Production of antibacterial compounds by parent and Ery ${ }^{r}$ mutant strains of S. griseus IFO13189 (c) and S. parvulus ATCC12434 (d). Strains of S. griseus and S. parvulus were inoculated onto TSB agar medium and GYM agar medium, which were incubated at $30^{\circ} \mathrm{C}$ for 24 and $60 \mathrm{~h}$, respectively. Agar plugs were cut from the plate and put on the assay plate that had been seeded with Bacillus subtilis ATCC6633, followed by $12 \mathrm{~h}$ incubation at $37^{\circ} \mathrm{C}$.

in the Ery ${ }^{\mathrm{r}}$ mutant SCE234 during the late growth phase (Supplementary Figure S1). In S. lividans, the Ery ${ }^{\mathrm{r}}$ SLE160 and SLE259 mutants, which exhibited a moderate level $\left(200 \mu \mathrm{g} \mathrm{ml}^{-1}\right)$ and a high level $\left(>500 \mu \mathrm{g} \mathrm{ml}^{-1}\right)$ of resistance to erythromycin, respectively, produced abundant ACT and RED. The Ery ${ }^{\mathrm{r}}$ mutant SLE299, with a level of resistance to erythromycin as high as the Ery ${ }^{\mathrm{r}}$ SLE259 mutant, exhibited lower antibiotic productivity compared with the parent strain 1326. These results suggest that many different types of erythromycin resistance mutations affect the ability of $S$. coelicolor A3(2) and S. lividans to produce antibiotics either positively or negatively.

Additionally, to assess the effects of a spontaneous mutation conferring erythromycin resistance on antibiotic production in other Streptomyces strains, a number of Ery ${ }^{\mathrm{r}}$ mutants ( $>100$ of each) were isolated from Streptomyces griseus IFO13189 (streptomycin producer), Streptomyces parvulus ATCC12434 (actinomycin producer), and Streptomyces antibioticus 3720 (actinomycin producer), and their ability to produce antibiotics were examined. In $S$. griseus and
S. parvulus, 14 and $24 \%$ of the Ery ${ }^{\mathrm{r}}$ mutants tested exhibited a significantly increased ability to produce antibiotics, although such a phenomenon was not found among the Ery ${ }^{\mathrm{r}}$ mutants of S. antibioticus 3720 (Supplementary Table S1). We do not know the reason why erythromycin resistance mutations were ineffective for antibiotic overproduction by $S$. antibioticus. Figures $1 \mathrm{c}$ and $\mathrm{d}$ show the antibiotic productivity by the representative overproducing Ery $^{\mathrm{r}}$ mutants isolated from S. griseus IFO13189 and $S$. parvulus ATCC12434 as examples. Thus, spontaneous mutations conferring erythromycin resistance in Streptomyces spp. effectively increase the productivity of antibiotics.

Surprisingly, we found that an ACT-overproducing Ery ${ }^{\mathrm{r}}$ mutant of S. lividans 1326, strain SLE259, produced abundant antibacterial compounds when it was grown on R4 agar medium. As shown in Figure $2 \mathrm{a}$, the antibacterial activity of strain SLE259 was clearly detected in both the presence and absence of calcium nitrate, suggesting that this activity could be due to multiple antibacterial compounds as well as calcium-dependent antibiotics. We then 
a

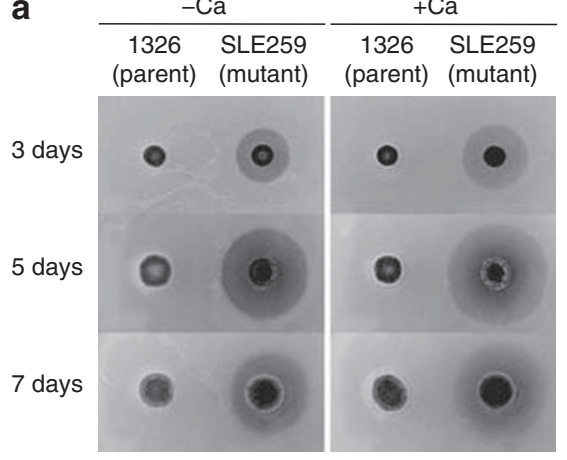

b

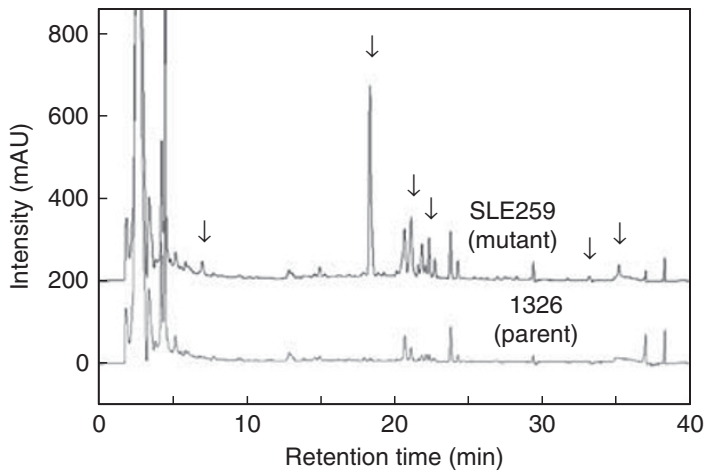

Figure 2 Detection and analysis of antibacterial compounds produced by Ery mutant of S. lividans 1326. (a) Production of antibacterial compounds by parent strain 1326 and Ery mutant SLE259. Strains were grown for 3-7 days on R4 agar medium, subsequently overlaid by Muellar Hinton agar medium (diluted two-fold) inoculated with Staphylococcus aureus 209P in the presence or absence of $12 \mathrm{~mm}$ calcium nitrate, and incubated at $37^{\circ} \mathrm{C}$ for $12 \mathrm{~h}$. (b) Comparative metabolic profiling of the culture extracts. Samples were prepared and analyzed by HPLC as described in experimental procedures (in supplementary information). The absorbance of eluate was monitored at 190-800 nm (maximum absorbance at any wavelength). The arrows indicate the peaks that were scarcely detected in the sample prepared from the parent strain.

compared the metabolic profile of the culture from the parent strain 1326 and Ery ${ }^{\mathrm{r}}$ mutant SLE259 by using HPLC with multi-wavelength monitoring (diode array detector). As a result, several peaks, which are indicated by arrows in Figure $2 \mathrm{~b}$, were identified only in the methanol-extractable fraction of the Ery ${ }^{\mathrm{r}}$ SLE259 mutant. The peaks were lacking or scarcely detectable in the fractions of the parent strain. These interesting phenomena were also observed when the Ery ${ }^{\mathrm{r}}$ SLE259 mutant was grown in R4 liquid medium (Supplementary Figures S2a and b). Although each peak detected in the samples of the Ery $^{\mathrm{r}}$ SLE259 mutant was not identified in this study, our results evidently show that the potential of S. lividans 1326 to produce secondary metabolites was dramatically activated by the acquisition of erythromycin resistance.

A mutation in the rplD and rplV genes, which encode ribosomal protein L4 and L22, respectively, confers resistance to erythromycin and other macrolide antibiotics in various bacteria. ${ }^{14-16} \mathrm{We}$, therefore, sequenced and compared these genes from the antibioticoverproducing Ery ${ }^{\mathrm{r}}$ mutants SCE234 and SLE259 to the parent strain of S. coelicolor A3(2) 1147 or S. lividans 1326. The mutation was, however, not detected in either $r p l D$ or $r p l V$ genes. As summarized in Table 1, the antibiotic-overproducing Ery ${ }^{\mathrm{r}}$ mutants were found to exhibit comparatively high resistance to other macrolide antibiotics and lincomycin, which may provide a clue to identifying the mutated gene of the Ery ${ }^{\mathrm{r}}$ mutants found in the present study.

In conclusion, we showed that antibiotic production in Streptomyces spp. is markedly increased through a spontaneous mutation conferring erythromycin resistance, although the mutated genes remain unknown. Most interestingly, an ACT-overproducing Ery ${ }^{\mathrm{r}}$ mutant of S. lividans 1326, strain SLE259, produced abundant antibacterial compounds that could include various substances as well as calcium-dependent antibiotics. Indeed, comparative metabolic profiling of the culture extracts using HPLC identified several substances that were present in the culture medium of strain SLE259 but not in the culture medium of the parent strain. We propose that the acquisition of erythromycin resistance in Streptomyces by certain spontaneous mutations could be an effective strategy for the development of their ability to produce secondary metabolites. Our next research goal is to identify the mutation responsible for erythromycin resistance and antibiotic overproduction, and to clarify the mechanisms by which this mutation elicits the ability of Streptomyces strains to produce secondary metabolites. We previously demonstrated, with antibiotic-overproducing ribosomal mutants of S. coelicolor A3(2) 1147 , the importance of maintaining a high level of protein synthesis at the late growth phase for activating antibiotic production. ${ }^{8-10}$ Although the mutated gene of antibiotic-overproducing Ery $^{\mathrm{r}}$ mutants isolated from S. coelicolor A3(2) 1147 and S. lividans 1326 was not identified, it is highly probable that ribosomal properties and concomitant protein synthesis activity was modified extensively by an erythromycin resistance mutation, because erythromycin targets ribosomal component.

Experimental procedures are detailed in the supplementary information online.

\section{ACKNOWLEDGEMENTS}

This study was performed through the Program for Dissemination of TenureTrack System funded by the Ministry of Education and Science, Japan, and partially supported by the Hokuto Bio-science Promotion Foundation. We are grateful to Drs Haifeng Hu and Yukinori Tanaka for discussions at the early stage of this study.

1 Kieser, T., Bibb, M. J., Buttner, M. J., Chater, K. F. \& Hopwood, D. A. Pratical Streptomyces Genetics. The John Innes Foundation, Norwich (2000).

2 Bentley, S. D. et al. Complete genome sequence of the model actinomycete Streptomyces coelicolor A3(2). Nature 417, 141-147 (2002).

3 Ikeda, H. et al. Complete genome sequence and comparative analysis of the industrial microorganism Streptomyces avermitilis. Nat. Biotechnol. 21, 526-531 (2003).

4 Ohnishi, Y. et al. Genome sequence of the streptomycin-producing microorganism Streptomyces griseus IFO 13350. J. Bacteriol. 190, 4050-4060 (2008).

5 Chiang, Y. M., Chang, S. L., Oakley, B. R. \& Wang, C. C. Recent advances in awakening silent biosynthetic gene clusters and linking orphan clusters to natural products in microorganisms. Curr. Opin. Chem. Biol. 15, 137-143 (2011).

6 Baltz, R. H. Strain improvement in actinomycetes in the postgenomic era. J. Ind. Microbiol. Biotechnol. 38, 657-666 (2011).

7 Ochi, K. et al. Ribosome engineering and secondary metabolite production. Adv. Appl. Microbiol. 56, 155-184 (2004).

8 Hosaka, T., Xu, J. \& Ochi, K. Increased expression of ribosome recycling factor is responsible for the enhanced protein synthesis during the late growth phase in an antibiotic-overproducing Streptomyces coelicolor ribosomal rpsL mutant. Mol. Microbiol. 61, 883-897 (2006).

9 Wang, G., Hosaka, T. \& Ochi, K. Dramatic activation of antibiotic production in Streptomyces coelicolor by cumulative drug resistance mutations. Appl. Environ. Microbiol. 74, 2834-2840 (2008).

10 Hosaka, T. et al. Antibacterial discovery in actinomycetes strains with mutations in RNA polymerase or ribosomal protein S12. Nat. Biotechnol. 27, 462-464 (2009).

11 Shima, J., Hesketh, A., Okamoto, S., Kawamoto, S. \& Ochi, K. Induction of actinorhodin production by $\operatorname{rps} L$ (encoding ribosomal protein S12) mutations that 
confer streptomycin resistance in Streptomyces lividans and Streptomyces coelicolor A3(2). J. Bacteriol. 178, 7276-7284 (1996).

$12 \mathrm{Hu}, \mathrm{H}$., Zhang, Q. \& Ochi, K. Activation of antibiotic biosynthesis by specified mutations in the $r p o B$ gene (encoding the RNA polymerase beta subunit) of Streptomyces lividans. J. Bacteriol. 184, 3984-3991 (2002).

13 Nishimura, K., Hosaka, T., Tokuyama, S., Okamoto, S. \& Ochi, K. Mutations in rsmG encoding a 16S rRNA methyltransferase, result in low-level streptomycin resistance and antibiotic overproduction in Streptomyces coelicolor A3(2). J. Bacteriol. 189, 3876-3883 (2007)
14 Wittmann, H. G. et al. Biochemical and genetic studies on two different types of erythromycin resistant mutants of Escherichia coli with altered ribosomal proteins. Mol. Gen. Genet. 127, 175-189 (1973).

15 Chittum, H. S. \& Champney, W. S. Ribosomal protein gene sequence changes in erythromycin-resistant mutants of Escherichia coli. J. Bacteriol. 176, 6192-6198 (1994)

16 Diner, E. J. \& Hayes, C. S. Recombineering reveals a diverse collection of ribosomal proteins L4 and L22 that confer resistance to macrolide antibiotics. J. Mol. Biol. 386, 300-315 (2009)

Supplementary Information accompanies the paper on The Journal of Antibiotics website (http://www.nature.com/ja) 